\title{
Evaluation of Cholera and Other Diarrheal Disease Surveillance System, Niger State, Nigeria-2012
}

\author{
Adebobola T. Bashorun*1, Anthony Ahumibe ${ }^{1}$, Saliman Olugbon ${ }^{1}$, Patrick Nguku ${ }^{1}$ and \\ Kabir Sabitu²
}

${ }^{1}$ Nigeria Field Epidemiology and Laboratory Training Program, FCT, Nigeria; ${ }^{2}$ Ahmadu Bello University, Zaria, Nigeria

\section{Objective}

To determine how the cholera and other diarrheal disease surveillance system in Niger state is meeting its surveillance objectives, to evaluate its performance and attributes and to describe its operation to make recommendations for improvement.

\section{Introduction}

Cholera causes frequent outbreaks in Nigeria, resulting in mortality. In 2010 and 2011, 41,936 cases (case fatality rate [CFR]-4.1\%) and 23,366 cases (CFR-3.2\%) were reported (1). Reported cases in Nigeria by week 26, 2012 was 309 (CFR-1.29\%) involving 20 Local Government Areas in 6 States. In Nigeria, there are currently eleven (11) States including Niger state at high risk for cholera/bloodless diarrhea outbreaks.

In 2011, Niger state had 2472 cholera cases (CFR-2\%) and 45,111 other diarrhea diseases cases, recorded in more than half of state Purpose of surveillance system is to ensure early detection of cholera and other diarrheal cases and to monitor trends towards evidencebased decision for management, prevention and control.

\section{Methods}

We conducted evaluation in July, 2012. We used CDC guideline on surveillance system evaluation (2001) as guide to assess operation, performance and attributes (2). We conducted key informant/in-depth interviews with stakeholders. We examined cholera action plans for preparedness and response, conducted laboratory assessment, extracted and analyzed cholera surveillance (2005-2012) for frequencies/proportions using Microsoft Excel. Thematic analysis was done for qualitative data. We shared findings with stakeholders at all levels.

\section{Results}

Surveillance system was setup for early detection and monitoring towards evidence-based decision. State government funds system. Case definition used is highly sensitive and is any patient aged 5 years or more who develops acute watery diarrhea, with/without vomiting. Though simple case definition, laboratory confirmation makes surveillance complex. A passive system, active during outbreaks; has formal and informal sources of information and part of Integrated Disease Surveillance and Response (IDSR) system and flow(fig.1). It takes 24-48 hours between outbreaks onset, confirmation and response.

Line list showed undefined/poorly labeled outcomes. Of 2472 cases in 20111320 (49\%) were found in line list. 2011 monthly data completeness was $75 \%$. So far in $2012,5(0.02 \%)$ of all diarrhea cases were cholera. System captures only age as sociodemographics.

Of 11 suspected cholera cases tested during 2011 epidemic, 7 confirmed as cholera (PPV-63\%). Of 3 rumours of cholera outbreaks (January 2011-July 2012), one (PPV-33\%) was true. Acceptability of system is high among all stakeholders interviewed. Timeliness of monthly reporting was $68.7 \%$ (Table 1 ).

Laboratory can isolate Vibro cholerae isolation but has no Cary Blair transport medium and cholera rapid test kits.

\section{Conclusions}

Evaluation revealed that surveillance system is meeting its objectives by early detection and response to cholera outbreaks. System is simple, stable, flexible, sensitive with poor data quality, low PPV, fair laboratory capacity and moderate timeliness. We recommended electronic and internet-based reporting for timeliness and data quality improvement; and provision of laboratory consumables.

Table 1. Summary of performance attributes of cholera surveillance system, Niger state, 2012.

\begin{tabular}{|c|c|c|c|c|c|c|}
\hline Performance attributes & $\begin{array}{c}\text { Excellent } \\
(>90 \%)\end{array}$ & $\begin{array}{c}\text { Very good } \\
(80-89 \%)\end{array}$ & $\begin{array}{c}\text { Good } \\
(70-79 \%)\end{array}$ & $\begin{array}{c}\text { Average } \\
(60-69 \%)\end{array}$ & $\begin{array}{c}\text { Fair } \\
(50-59 \%)\end{array}$ & $\begin{array}{c}\text { Poor } \\
(<50 \%)\end{array}$ \\
\hline Simplicity & & $\& \# 9679 ;$ & & & & \\
\hline Flexibility & $\& \# 9679 ;$ & & & & & \\
\hline Data Quality & & & & & $\& \# 9679 ;$ & \\
\hline Acceptability & $\& \# 9679 ;$ & & & & & \\
\hline Sensitivity & & $\& \# 9679 ;$ & & & & \\
\hline Positive Predictive Value & & & & $\& \# 9679 ;$ & & \\
\hline Representativeness & & & & $\& \# 9679 ;$ & & \\
\hline Timeliness & & & $\& \# 9679 ;$ & & & \\
\hline Stability & & $\& \# 9679 ;$ & & & & \\
\hline
\end{tabular}

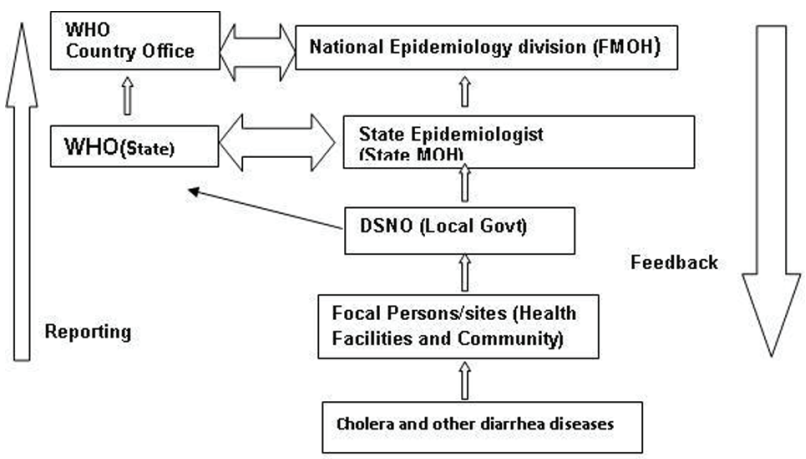

Keywords

Surveillance; Evaluation; Cholera; Nigeria

\section{Acknowledgments}

Niger State Ministry of Health

\section{References}

1. Federal Ministry of Health, Nigeria Weekly Epidemiology Reports, NCDC/Federal Ministry of Health - Nigeria Volume 1 No 1-52. 2011

2. Centers for Disease Control and Prevention. Updated guidelines for evaluating public health surveillance systems: recommendations from the guidelines working group. MMWR 2001;50 (No. RR-13)

\section{*Adebobola T. Bashorun}

E-mail: bashogee@yahoo.com 